\title{
The Kinematics Analysis of Heald Selecting Mechanism of Rotary Electronic Dobby
}

\author{
Hongbin Yu1,2, Xiangpeng Zhao1, Honghuan Yin1, Hongyu Shao ${ }^{3}$ \\ ${ }^{1}$ College of Mechanical Engineering, Tianjin Polytechnic University, Tianjin, China \\ ${ }^{2}$ Key Laboratory of Advanced Mechatronics Equipment Technology, Tianjin Polytechnic University, Tianjin, China \\ ${ }^{3}$ College of Mechanical Engineering, Tianjin University, Tianjin, China \\ Email: yuhongbin@tiangong.edu.cn
}

How to cite this paper: Yu, H.B., Zhao, X.P., Yin, H.H. and Shao, H.Y. (2021) The Kinematics Analysis of Heald Selecting Mechanism of Rotary Electronic Dobby. Open Journal of Applied Sciences, 11, 93-102. https://doi.org/10.4236/ojapps.2021.111007

Received: December 27, 2020

Accepted: January 24, 2021

Published: January 27, 2021

Copyright (c) 2021 by author(s) and Scientific Research Publishing Inc. This work is licensed under the Creative Commons Attribution International License (CC BY 4.0).

http://creativecommons.org/licenses/by/4.0/

\begin{abstract}
In order to improve the reliability of the mechanical movement of the rotary electronic dobby, the kinematics analysis of the heald selection mechanism is carried out and the simulation is carried out with Matlab. Firstly, the operation mechanism of the heald selection mechanism is analyzed in detail. The conjugate cam is mapped. The cam profile curve is fitted with cubic spline interpolation. Secondly, based on the overall analysis method and the complex vector method, the kinematics analysis of the key components after the high pair low generation is performed, and the angular displacement and angular velocity of each component are calculated with the rotation of the active cam. Finally, the movement curve diagram is drawn with Matlab, which lays the foundation for the dynamic analysis and in-depth study of the selection mechanism in the future.
\end{abstract}

\section{Keywords}

Heald Selection Mechanism, Substitute Equivalent Linkage Instead of Higher Pair, Complex Number Vector Method, The Kinematics Analysis

\section{Introduction}

As the most advanced high-speed shedding device [1], the rotary dobby machine is widely used in many new looms. Its function is to convert the information stored in the computer into electrical signals to control the release and attraction of the electromagnet, and then choose to control the up and down movement of the heald frame.

The rotary dobby is composed of heald selection mechanism, heddle lifting mechanism, electronic control equipment module, etc. The heald selection me- 
chanism is the core control mechanism of the dobby [2], which determines the lifting sequence of the heald frame according to its control sequence, and its mechanical motion process requires a very high accuracy. In order to ensure the precise coordination between the heddle selection mechanism and the heddle lifting mechanism and avoid collisions, it is of great significance to analyze the kinematics of the heddle selection mechanism.

At present, for the heddle selection mechanism of the dobby machine, Shen Yi et al. [3] [4] carried out a three-dimensional modeling of the structure of the heddle selection, designed and analyzed the working principle and control process of the dobby machine. The conjugate cam is kinematically designed, and the conjugate cam is derived using polar coordinate theory, and the conjugate cam is reconstructed. The commonly used methods for the motion analysis of the planar linkage mechanism are graphic method and analytical method [5], the graphic method is intuitive and vivid, but for high-speed dobby machines, the accuracy is not high. The analytical method is fast and efficient. The overall method in the analytical method can accurately establish the functional relationship between the overall mechanism size parameters and the motion parameters. This article uses complex vector method to analyze the kinematics of the selection mechanism.

As the society has higher and higher requirements for production efficiency, the speed, accuracy and reliability of the rotary dobby have been continuously improved. As the key control mechanism of the dobby, the heddle selection mechanism is of great significance to accurately establish the motion model of the rotary dobby and conduct in-depth kinematics analysis on it.

For this reason, this article uses the high pair low generation method [6] to establish a kinematic model under the premise of precise coordination between the selection mechanism and the lifting mechanism, and the three-coordinate mapping of the conjugate cam [7] obtains a series of discrete points. Cubic spline interpolation method [8] fits the cam profile curve, and uses the analytical method to analyze the kinematics of the plane mechanism, establishes the mathematical model of the mechanism, and uses Matlab [9] [10] to calculate the angle of every member graph of displacement, angular velocity and angular acceleration.

The selection system includes a selection cam and a selection control mechanism, consisting of a conjugate cam 5 , a roller 6 , an auxiliary swing $\operatorname{arm} 7$, a main swing arm 8 , an iron suction swing lever 10 , a right signal swing arm 12 , and a left signal swing arm 13. The composition is shown in Figure 1. The rotating speed change mechanism in the heald lifting system converts the uniform motion into the variable speed rotation of the main shaft 1, drives the drive disc 2 to rotate, and then the heald frame moves up and down. In the heald selection system, the power input is the heald selection conjugate cam fixedly connected with the large disc, the main swing arm 8 and the auxiliary swing arm 7 swing along the conjugate cam, and the iron-absorbing swing rod and the main swing arm are fixedly connected by the shaft together, with the swing of the main swing 


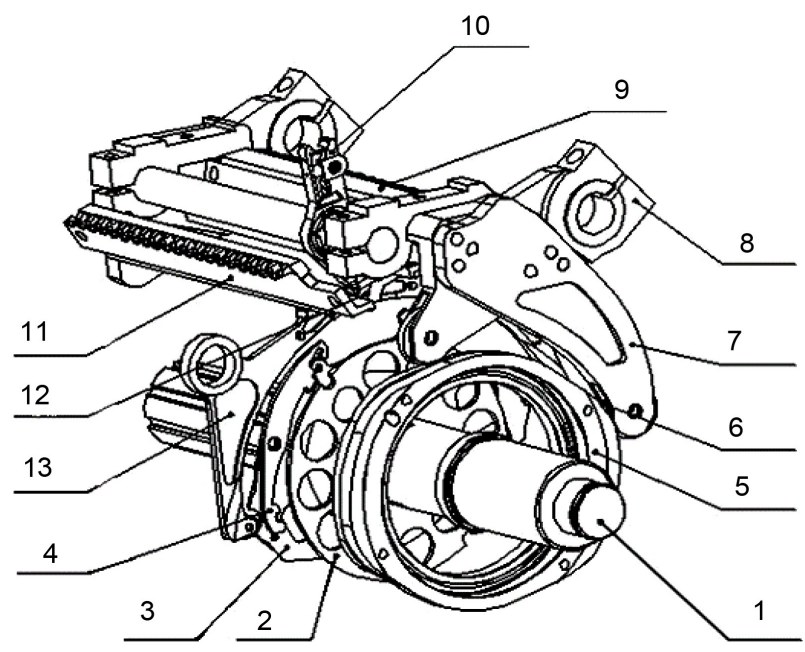

Figure 1. Structure diagram of selection system. 1-Main shaft, 2-Drive disc, 3-Lifting heddle plate, 4-Clutch claw, 5-Selection heddle conjugate cam, 6-Roller, 7-Auxiliary swing arm, 8-Main swing arm, 9-Right baffle, 10-Swing lever with iron absorption, 11-Left baffle, 12-Swing arm with right signal, 13-Swing arm with left signal.

arm, the release and pull-in of the electromagnet determines whether the iron-absorbing swing rod is in contact with the right signal swing arm 12 and the left signal swing arm 13, and the iron-absorbing swing rod contacts the signal swing arm. The shaft rotates, and under the action of the clutch pawl 4, the driving disc 2 and the heald lifting disc 3 become a whole, thereby completing the selection of the heald frame. In order to realize the precise control of the heald frame by the heald selection system and avoid the mechanical collision between the heald selection system and the heald lifting system, a safe corner must exist.

\section{Material and Methods}

Since the movement of the right signal swing arm and the left signal swing arm are similar, the left signal swing arm is selected for kinematic analysis, and the instantaneous state of the mechanism is simplified. The mechanism selects the kinematics of the comprehensive system according to the method of high pair and low generation of the mechanism. The model is simplified into a plane link mechanism model, the mechanism's high and low degrees of freedom, instantaneous speed, and instantaneous acceleration remain unchanged. Figure 2 is a schematic diagram of the working principle of the rotary dobby. The model mechanism is a combination of a crank rocker mechanism and a swing guide rod mechanism.

Measure the actual profile of the conjugate cam, use a three-coordinate measuring instrument to map the cam, as shown in Figure 3, to obtain a set of discrete points on the cam profile.

After the cam high pair low is replaced, the cubic spline interpolation method 


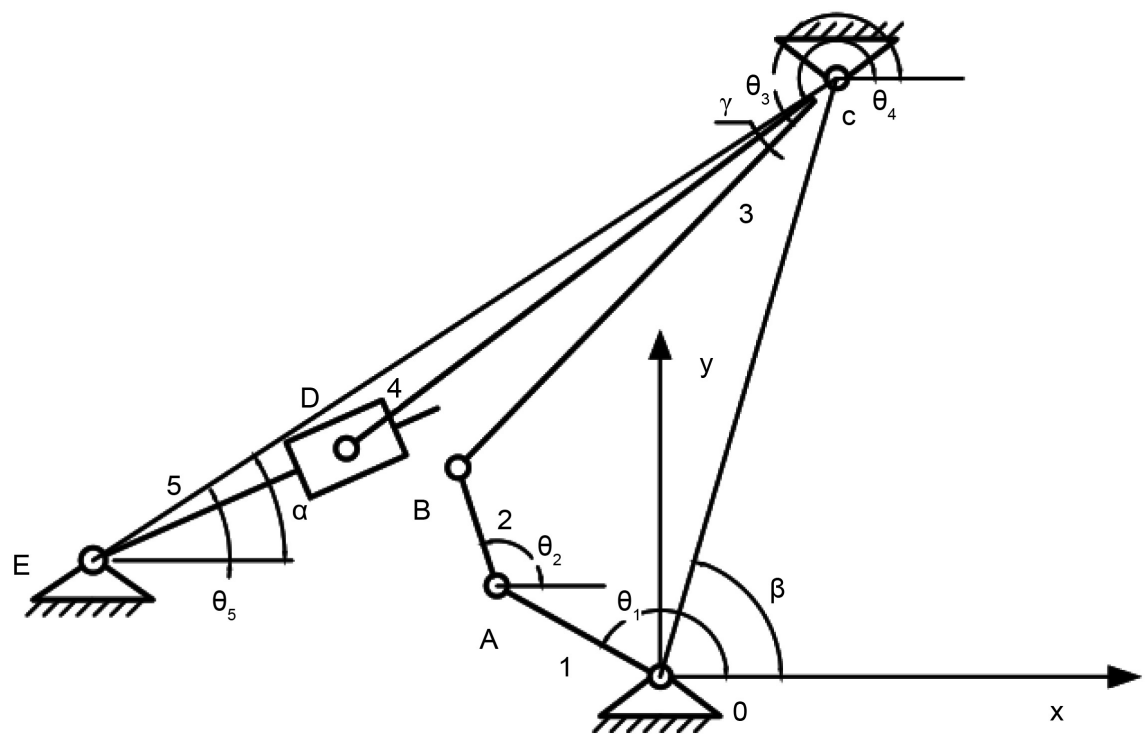

Figure 2. The movement diagram of the heald selection mechanism of the rotary dobby machine. 1-Original moving part, 2-Virtual rod, 3-Main swing arm, 4-Iron-absorbing swing rod, 5-Left signal swing arm.

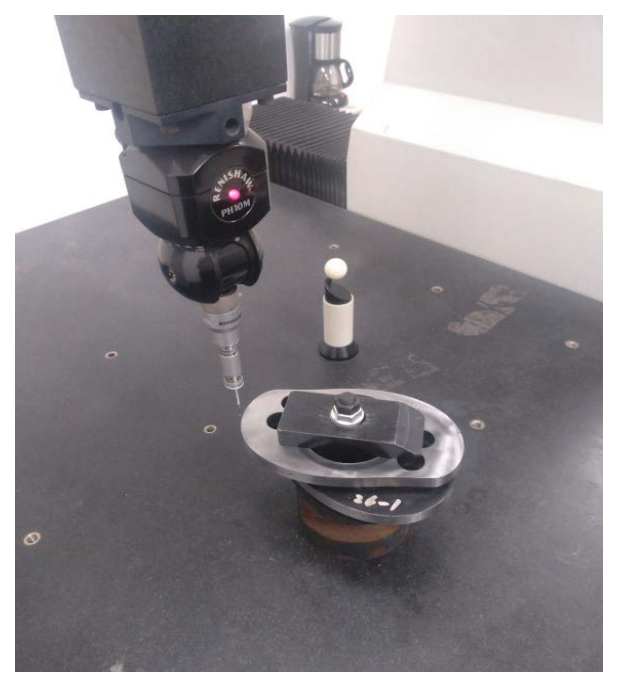

Figure 3. Three-coordinate measurement.

is used to solve the curvature radius of the plane cam whose cam profile is discrete points, as shown in Figure 4.

Calculation formula of radius of curvature

$$
\rho=\frac{\left[\left(x^{\prime}\right)^{2}+\left(y^{\prime}\right)^{2}\right]^{3 / 2}}{x^{\prime} y^{\prime \prime}-x^{\prime \prime} y^{\prime}}
$$

where $x^{\prime}, y^{\prime}, x^{\prime \prime}, y^{\prime \prime}$ can be obtained by cubic spline interpolation, the coordinates of the center of curvature $A_{j}$

$$
\left\{\begin{array}{l}
x_{A_{j}}=x_{B_{j}}-\rho_{B} \cos \alpha_{B_{j}} \\
y_{A_{j}}=x_{B_{j}}-\rho_{B_{j}} \sin \alpha_{B_{j}}
\end{array}\right.
$$




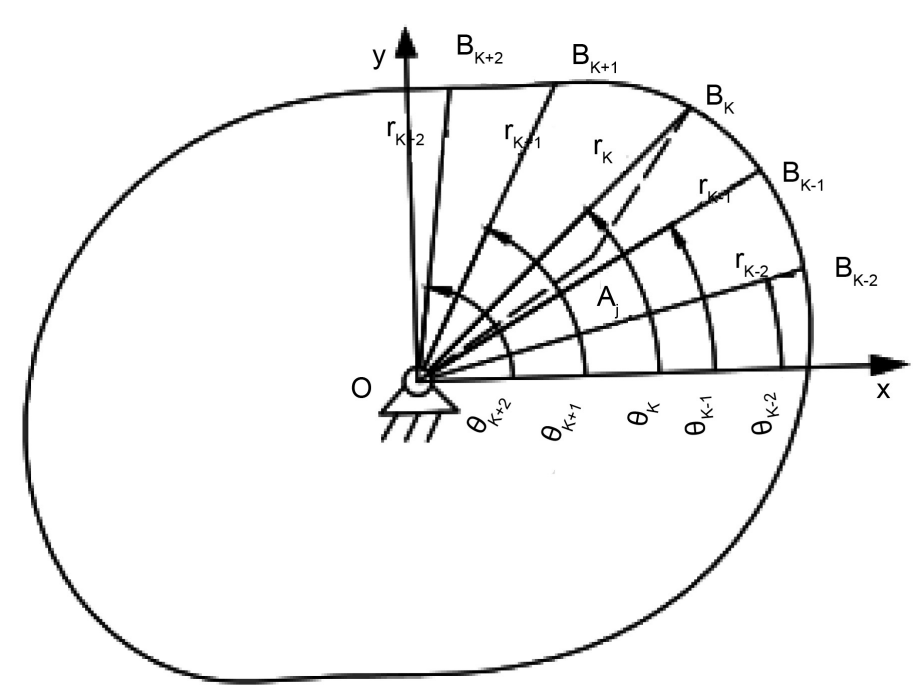

Figure 4. The center of curvature of the conjugate cam.

The rod length of the members $O A_{j}, B_{j} A_{j}$ is $l_{O A_{j}}, l_{B_{j} A_{j}}$ is

$$
\begin{gathered}
l_{O A_{j}}=\sqrt{\left(x_{A_{j}}-x_{O}\right)^{2}+\left(y_{A_{j}}-y_{O}\right)^{2}} \\
l_{B_{j} A_{j}}=\sqrt{\left(x_{A_{j}}-x_{B_{j}}\right)^{2}+\left(y_{A_{j}}-y_{B_{j}}\right)^{2}}
\end{gathered}
$$

\subsection{Displacement Analysis}

Kinematics analysis of crank rocker mechanism and swing guide rod mechanism respectively, and the vector equation is obtained from the kinematic model

$$
\begin{gathered}
O A+A B=O C+C B \\
E C+C D=E D
\end{gathered}
$$

Write Formula (5) in plural form

$$
l_{O A} \mathrm{e}^{i \theta_{1}}+l_{A B} \mathrm{e}^{i \theta_{2}}=l_{O C} \mathrm{e}^{i \beta}+l_{C B} \mathrm{e}^{i \theta_{3}}
$$

The real and imaginary parts of Formula (7) are respectively equal to obtain

$$
\begin{gathered}
l_{O A} \cos \theta_{1}+l_{A B} \cos \theta_{2}=l_{O C} \cos \beta+l_{C B} \cos \theta_{3} \\
l_{O A} \sin \theta_{1}+l_{A B} \sin \theta_{2}=l_{O C} \sin \beta+l_{C B} \sin \theta_{3}
\end{gathered}
$$

And then get

$$
l_{A B}^{2}=\left(l_{O C} \cos \beta+l_{C B} \cos \theta_{3}-l_{O A} \cos \theta_{1}\right)^{2}+\left(l_{O C} \sin \beta+l_{C B} \sin \theta_{3}-l_{O A} \sin \theta_{1}\right)^{2}
$$

To facilitate the solution of $\theta_{3}$, Formula (10) can be rewritten as the following equation

$$
A \sin \theta_{3}+B \cos \theta_{3}+C=0
$$

where: $A=2 l_{C B} l_{O C} \sin \beta-2 l_{O A} l_{C B} \sin \theta_{1} ; \quad B=2 l_{C B} l_{O C} \cos \beta-2 l_{O A} l_{C B} \cos \theta_{1}$; $C=l_{O C}^{2}+l_{C B}^{2}+l_{O A}^{2}-l_{A B}^{2}-2 l_{O A} l_{O C}\left(\cos \theta_{1} \cos \beta+\sin \theta_{1} \sin \beta\right)$

$$
\theta_{3}=2 \arctan \frac{A \pm \sqrt{A^{2}+B^{2}-C^{2}}}{B-C}
$$




$$
\theta_{2}=\arctan \frac{l_{O C} \sin \beta+l_{C B} \sin \theta_{3}-l_{O A} \sin \theta_{1}}{l_{O C} \cos \beta+l_{C B} \cos \theta_{3}-l_{O A} \cos \theta_{1}}
$$

In the formula, write Formula (6) in plural form

$$
l_{E C} \mathrm{e}^{i \alpha}+l_{C D} \mathrm{e}^{i \theta_{4}}=l_{E D} \mathrm{e}^{i \theta_{5}}
$$

The real part and imaginary part of Formula (14) are respectively equal to obtain

$$
\begin{aligned}
l_{E C} \cos \alpha+l_{C D} \cos \theta_{4} & =l_{E D} \cos \theta_{5} \\
l_{E C} \sin \alpha+l_{C D} \sin \theta_{4} & =l_{E D} \sin \theta_{5}
\end{aligned}
$$

The same can be obtained

$$
\theta_{5}=\arctan \frac{l_{E C} \sin \alpha+l_{C D} \sin \theta_{4}}{l_{E C} \cos \alpha+l_{C D} \cos \theta_{4}}
$$

In triangular $\mathrm{CDE}$, from the law of cosines

$$
l_{E D}=\sqrt{l_{E C}^{2}+l_{C D}^{2}-2 l_{E C} l_{C D} \cos \left(\theta_{4}-\alpha\right)}
$$

Can be obtained from the assembly relationship

$$
\theta_{4}=\theta_{3}-\gamma
$$

So far, the change rule of the position of each rod of the selection mechanism with the rotation angle $\theta_{1}$ of the conjugate cam can be obtained.

\subsection{Speed Analysis}

Deriving Formula (7) with respect to time can be obtained

$$
l_{O A} \omega_{1} \mathrm{e}^{i\left(\theta_{1}+\frac{\pi}{2}\right)}+l_{A B} \omega_{2} \mathrm{e}^{i\left(\theta_{2}+\frac{\pi}{2}\right)}=l_{C B} \omega_{3} \mathrm{e}^{i\left(\theta_{3}+\frac{\pi}{2}\right)}
$$

The real part and imaginary part of Formula (20) are respectively equal to obtain

$$
\begin{gathered}
-l_{O A} \omega_{1} \sin \theta_{1}-l_{A B} \omega_{2} \sin \theta_{2}=-l_{C B} \omega_{3} \sin \theta_{3} \\
l_{O A} \omega_{1} \cos \theta_{1}+l_{A B} \omega_{2} \cos \theta_{2}=l_{C B} \omega_{3} \cos \theta_{3}
\end{gathered}
$$

It can be solved by Formulas (21) and (22)

$$
\begin{gathered}
\omega_{2}=\frac{-l_{O A} \sin \left(\theta_{1}-\theta_{3}\right)}{l_{A B} \sin \left(\theta_{2}-\theta_{3}\right)} \cdot \omega_{1} \\
\omega_{4}=\omega_{3}=\frac{l_{O A} \sin \left(\theta_{1}-\theta_{2}\right)}{l_{C B} \sin \left(\theta_{3}-\theta_{2}\right)} \cdot \omega_{1}
\end{gathered}
$$

Derivative Formula (14) with respect to time can be obtained.

$$
l_{C D} \omega_{4} \mathrm{e}^{i\left(\theta_{4}+\frac{\pi}{2}\right)}=l_{E D} \omega_{5} \mathrm{e}^{i\left(\theta_{5}+\frac{\pi}{2}\right)}+v_{r} \mathrm{e}^{i \theta_{5}}
$$

The relative velocity of component 5 is $v_{r}$.

The real part and imaginary part of Formula (25) are respectively equal to obtain

$$
-l_{C D} \omega_{4} \sin \theta_{4}=-l_{E D} \omega_{5} \sin \theta_{5}+v_{r} \cos \theta_{5}
$$




$$
l_{C D} \omega_{4} \cos \theta_{4}=l_{E D} \omega_{5} \cos \theta_{5}+v_{r} \sin \theta_{5}
$$

Rotate the $x E y$ coordinate system around point $E$ by angle $\theta_{5}$ Then by Formulas (26), (27) we can get

$$
\begin{aligned}
& \omega_{5}=\frac{l_{C D} \omega_{4} \cos \left(\theta_{4}-\theta_{5}\right)}{l_{E D}} \\
& v_{r}=-l_{C D} \omega_{4} \sin \left(\theta_{4}-\theta_{5}\right)
\end{aligned}
$$

\subsection{Acceleration Analysis}

Derivation of Formula (20) with respect to time can be obtained

$$
l_{O A} \omega_{1}^{2} \mathrm{e}^{i\left(\theta_{1}+\pi\right)}+l_{A B} \omega_{2}^{2} \mathrm{e}^{i\left(\theta_{2}+\pi\right)}+l_{A B} \alpha_{2} \mathrm{e}^{i\left(\theta_{2}+\frac{\pi}{2}\right)}=l_{C B} \omega_{3}^{2} \mathrm{e}^{i\left(\theta_{3}+\pi\right)}+l_{C B} \alpha_{3} \mathrm{e}^{i\left(\theta_{3}+\frac{\pi}{2}\right)}
$$

The real and imaginary parts of Formula (30) are respectively equal to obtain

$$
\begin{aligned}
& l_{O A} \omega_{1}^{2} \cos \theta_{1}+l_{A B} \omega_{2}^{2} \cos \theta_{2}+l_{A B} \alpha_{2} \sin \theta_{2}=l_{C B} \omega_{3}^{2} \cos \theta_{3}+l_{C B} \alpha_{3} \sin \theta_{3} \\
& -l_{O A} \omega_{1}^{2} \sin \theta_{1}-l_{A B} \omega_{2}^{2} \sin \theta_{2}+l_{A B} \alpha_{2} \cos \theta_{2}=-l_{C B} \omega_{3}^{2} \sin \theta_{3}+l_{C B} \alpha_{3} \cos \theta_{3}
\end{aligned}
$$

By Formula (31), (32) can be solved

$$
\begin{aligned}
& \alpha_{3}=\frac{l_{O A} \omega_{1}^{2} \cos \theta_{1}-\theta_{2}+l_{A B} \omega_{2}^{2}-l_{C B} \omega_{3}^{2} \cos \left(\theta_{3}-\theta_{2}\right)}{l_{C B} \sin \left(\theta_{3}-\theta_{2}\right)} \\
& \alpha_{2}=\frac{l_{O A} \omega_{1}^{2} \cos \theta_{1}-\theta_{2}+l_{A B} \omega_{2}^{2} \cos \left(\theta_{3}-\theta_{2}\right)-l_{C B} \omega_{3}^{2}}{l_{A B} \sin \left(\theta_{3}-\theta_{2}\right)}
\end{aligned}
$$

Derivation of Formula (25) with respect to time can be obtained

$$
l_{C D} \omega_{4}^{2} \mathrm{e}^{i\left(\theta_{4}+\pi\right)}=2 v_{r} \omega_{5} \mathrm{e}^{i\left(\theta_{5}+\frac{\pi}{2}\right)}+l_{E D} \omega_{5}^{2} \mathrm{e}^{i\left(\theta_{5}+\pi\right)}+l_{E D} \alpha_{5} \mathrm{e}^{i\left(\theta_{5}+\frac{\pi}{2}\right)}+a_{r} \mathrm{e}^{i \theta_{5}}
$$

The same can be obtained

$$
\begin{gathered}
a_{r}=l_{E D} \omega_{5}^{2}-l_{C D} \omega_{4}^{2} \cos \left(\theta_{4}-\theta_{5}\right) \\
\alpha_{5}=-\left[2 v_{r} \omega_{5}+l_{C D} \omega_{4}^{2} \sin \left(\theta_{4}-\theta_{5}\right)\right] / l_{E D}
\end{gathered}
$$

\section{Results and Discussion}

The geometric symbols and physical parameters of the heddle selection mechanism of a certain type of rotary dobby machine are shown in Table 1 . The speed of the large disk is set to $100 \mathrm{r} / \mathrm{min}$, in order to realize the heddle selection control function, and the signal swing arm and the heddle lifting plate do not collide. Set the safety angle to $15^{\circ}$.

Through Matlab software programming calculation, the angular displacement, angular velocity and angular acceleration curves of the iron-absorbing pendulum are obtained, as shown in Figures 5-7.

It can be seen from Figures 5-7 that the angular displacement of the iron-absorbing pendulum is within the safe corner $15^{\circ}$, the angular velocity and angular acceleration meet the requirements of the motion characteristics, and the angular acceleration curve is smoother. 
Table 1. Description of geometric symbols and physical parameters of selection mechanism.

\begin{tabular}{cc}
\hline Structural parameters & Description \\
\hline$i$ & Component number \\
$\theta_{i}$ & The included angle between the $i$ component and the positive $x$-axis \\
$L_{B d} / \mathrm{mm}$ & 137.9 \\
$L_{C D} / \mathrm{mm}$ & 137.9 \\
$L_{O} / \mathrm{mm}$ & 204.1 \\
$L_{E d} / \mathrm{mm}$ & 185.8 \\
$\alpha /\left(^{\circ}\right)$ & 31.2 \\
$\beta /\left(^{\circ}\right)$ & 69.7 \\
$\gamma /\left(^{\circ}\right)$ & 20.3 \\
\hline
\end{tabular}

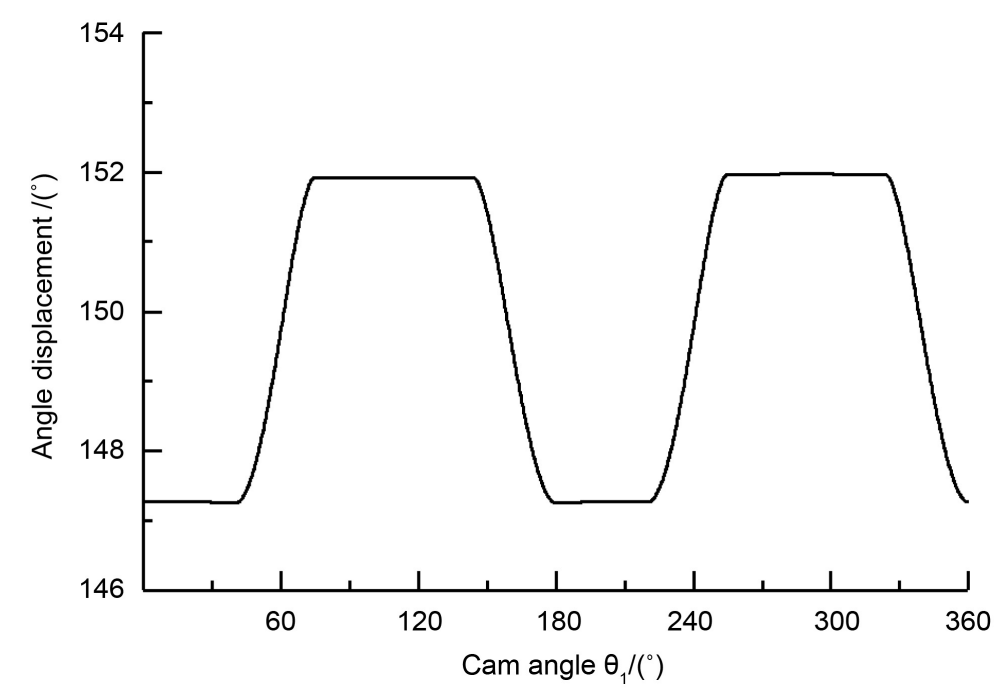

Figure 5. Angular displacement of the iron-absorbing pendulum.

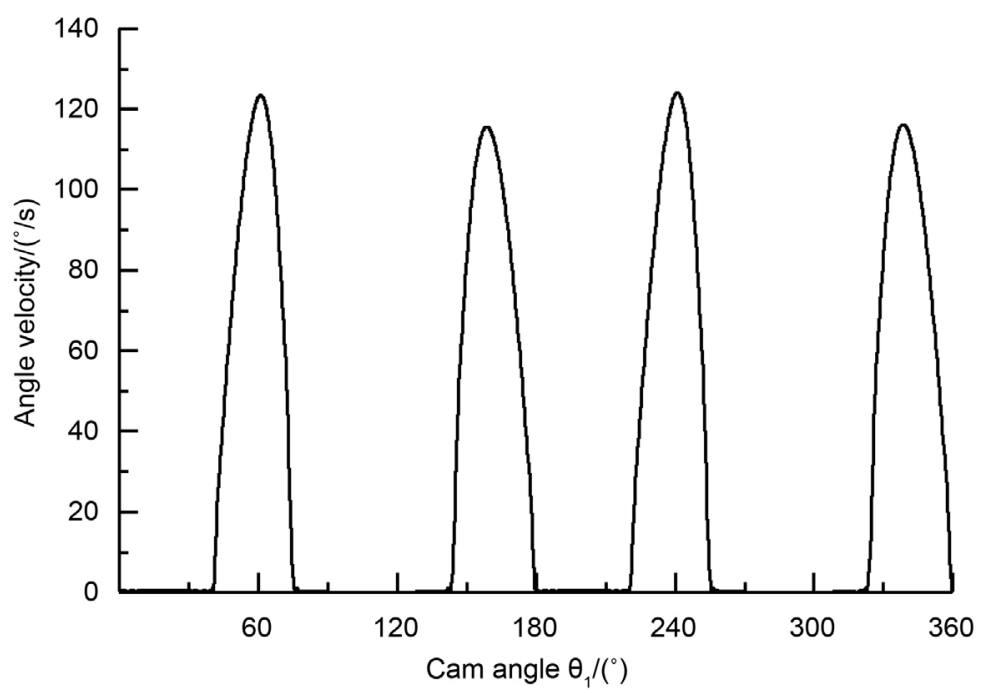

Figure 6. Angular velocity of iron-absorbing pendulum. 


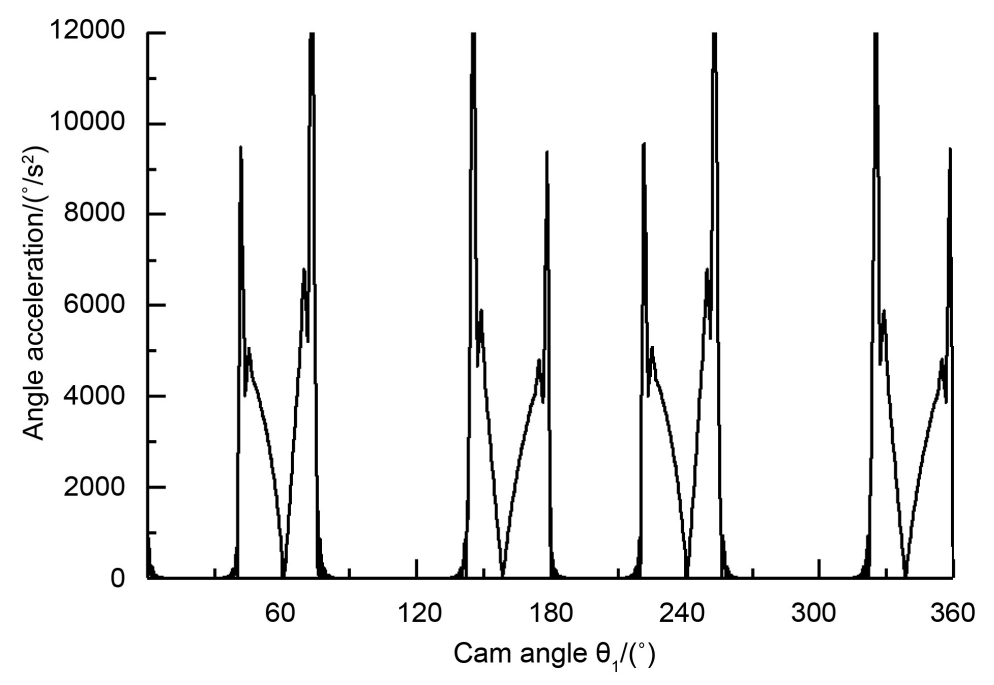

Figure 7. Angular acceleration of iron-absorbing pendulum.

\section{Conclusion}

The operating principle of the rotary dobby machine selection mechanism is analyzed deeply. Build a kinematic model, and use the three-coordinate instrument to measure the contour of the conjugate cam to obtain discrete points. The kinematics analysis of the heald selection mechanism is carried out and the simulation is carried out with Matlab. Determine the law of change of the angular displacement and angular velocity of every component accurately, locate the motion trajectory of every component accurately, and avoid interference between the selection mechanism and the lifting mechanism, providing a theoretical basis for whether the speed and acceleration meet the requirements, and providing a basis for future kinetic analysis.

\section{Conflicts of Interest}

The authors declare no conflicts of interest regarding the publication of this paper.

\section{References}

[1] Eren, R., Ozkan, G. and Turhan, Y. (2008) Kinematics of Rotary Dobby and Analysis of Heald Frame Motion in Weaving Process. Textile Research Journal, 78, 1070-1079. https://doi.org/10.1177/0040517507083549

[2] Umair, M., Nawaab, Y., Malik, M.H., et al. (2015) Development and Characterization of Three-Dimensional Woven-Shaped Preforms and Their Associated Composites. Journal of Reinforced Plastics and Composites, 34, 2018-2028. https://doi.org/10.1177/0731684415608958

[3] Eren, R., Ozkan, G. and Karahan, M. (2005) Comparison of Heald Frame Motin Generated by Rotary Dobby and Crank \& Camsheddig Motions. Fibraes \& Textiles in Eastern Europe, 13, 78-83.

[4] Guha, A., Amarnath, C., Issac, K.K., et al. (2009) Linkage Driven Dobby with Dwel. Textile Research Journal, 79, 804-809. https://doi.org/10.1177/0040517507087673

[5] Shao, J.C., Hamano, Y. and Fuller, M.D. (2007) Representation of the Main Geo- 
magnetic Field Using Maxwell's Theory of Poles. Geophysical Journal International, 169, 91-99. https://doi.org/10.1111/j.1365-246X.2006.03300.x

[6] Yu, X. and Lan, Z. (2008) The Application of High Pair and Low Generation in the Motion Analysis of Planar Cam Mechanism with Roller Translating Follower. Journal of Fuzhou University (Natural Science Edition), No. 5, 709-713.

[7] Ji, X. (2008) Analysis of Surveying and Mapping of Planar Conjugate Cam Mechanism. Textile Machinery, No. 6, 46-48.

[8] Yu, X. and Lan, Z. (2008) Application of Cubic Spline Interpolation in Solving the Radius of Curvature of Plane Cam Profile. Mechanical Transmission, No. 1, 50-51+111.

[9] Zhang, R. (2004) Proficient in MATLAB6.5. China Water Power Press, Beijing.

[10] Zuo, W., Deng, Y. and Wei, B. (2011) Kinematics Analysis and Dynamic Simulation of Planar Linkage Mechanism Based on MATLAB. Journal of Hubei University of Technology, 26, 84-86. 